Review

Plant Genetics

\title{
Flowering process in soybean under water deficit conditions: A review on genetic aspects
}

\author{
Mayla Daiane Correa Molinari ${ }^{1,2^{*}}$ (D), Renata Fuganti-Pagliarini ${ }^{*}$ (D), Daniel de Amorim Barbosa ${ }^{1,2}$, \\ Silvana Regina Rockenbach Marin², Daniel Rockenbach Marin², Elíbio Leopoldo Rech ${ }^{3}$, \\ Liliane Marcia Mertz-Henning ${ }^{2}$ and Alexandre Lima Nepomuceno ${ }^{2}$ \\ ${ }^{1}$ Universidade Estadual de Londrina, Departamento de Biologia Geral, Londrina, PR, Brazil. \\ ${ }^{2}$ Embrapa Soja, Londrina, PR, Brazil. \\ ${ }^{3}$ Embrapa Recursos Genéticos e Biotecnologia, Instituto Nacional de Ciência e Tecnologia em Biologia \\ Sintética, Brasília, DF, Brazil.
}

\begin{abstract}
Soybean is a key crop in many countries, being used from human food to the animal industry due to its nutritional properties. Financially, the grain chain moves large sums of money into the economy of producing countries. However, like other agricultural commodities around the world, it can have its final yield seriously compromised by abiotic environmental stressors, like drought. As flowers imply in pods and in grains inside it to minimize damages caused by water restriction, researchers have focused on understanding flowering-process related genes and their interactions. Here a review dedicated to the soybean flowering process and gene network involved in it is presented, describing gene interactions and how genes act in this complex mechanism, also ruled by environmental triggers such as daylight and circadian cycle. The objective was to gather information and insights on the soybean flowering process, aiming to provide knowledge useful to assist in the development of drought-tolerant soybean lines, minimizing losses due to delays or anticipation of flowering and, consequently, restraining financial and productivity losses.
\end{abstract}

Keywords: Water deficit, Glycine max, climate changes, flowers, pods.

Received: January 24, 2021; Accepted: September 30, 2021.

\section{Introduction}

Soybean is one of the most planted commodities in the world. It is a source of animal and human food due to its various nutrient and functional compounds. Also, the grain is widely used in the oil and animal feed industry, employing thousands of people, and moving millions of dollars annually. From 2015 to 2019, the USA was in the first place in the ranking of global soybean producers (USDA, 2021). In 2020, Brazil outstripped the United States, producing 124 million metric tons, production about 3\% higher than the USA, taking the leadership position in the rank of producers and remaining as a main player in 2020/2021 crop season with about 137 million metrics (CONAB, 2021; USDA, 2021).

Despite high productivity, in crop seasons affected by abiotic conditions such as drought, yield can be seriously impaired, leading to significant economic losses. If the lack of water occurs in sensitive developmental periods such as in flowering and pod filling, the impact can be huge, downsizing productivity between 78 to $97 \%$ when compared to good-water crop seasons (Ferreira, 2016). Data collected from 1976/77 to $2013 / 14$ crop seasons in Brazil indicated up to US\$79,62 billion in financial losses due to water deficit (Ferreira, 2016).

Send correspondence to Renata Fuganti-Pagliarini. Embrapa Soja, Rodovia Carlos João Strass, acesso Orlando Amaral 231, Warta, 86001-970, Londrina, PR, Brazil. E-mail: renatafuganti@ gmail.com.

*These authors contributed equally to the article.
Thus, drought can jeopardize the main hope of any soybean producer, which is to have his crop filling as many pods as possible. However, it begins with the number of nodes followed by the number of flowers set. The greater the number of nodes and branches, the greater the flower-bearing potential. In other words, the more physically spread-out flowers are on a plant, the greater the final production of pods. However, under drought conditions, flowers and pods abortion can occur, dropping final yields numbers. In pods, the period of latter pod formation is particularly critical, as flowering has ceased, there is no further compensation for lost pods. Usually, vulnerability to abortion under water deficit is higher in younger pods when compared to older pods/ seeds. Depending on soybean genetics, seed size can still be compensated, if rain occurs after R5 (pod fill), which can reduce yield losses. Furthermore, seed number per pod and seed size can also be impaired but to a minor magnitude than pod numbers (Desclaux et al., 2000). Water deficit-stressed plants frequently mature earlier, shortening the grain filling period, and consequently reducing seed weight and final yield (Licht et al., 2013).

Therefore, considering the current climate scenario and future projections pointing out that the type, frequency, and intensity of extreme events should increase as Earth's climate changes (IPCC, 2021), the comprehension of the flowering mechanisms and the genes involved in this pathway, could give necessary information and be an alternative tool to minimize losses, in the next decades, since the switching from the vegetative to the reproductive phase relates closely to 
the success in crop productivity (Cai et al., 2020). Moreover, with the currently available editing tools, these genes can be candidates in the development of new soybean cultivars aiming to eliminate or mitigate drought- stress, ensuring that a great number of flowers/pods would survive to reduce yield losses.

\section{Gene network involved in flowering in soybean - studies involving mutation and/or overexpression}

Soybean is a short-day dicot plant (i.e., long nights or dark periods for flowering induction), meaning that the cue for floral induction depends on soybean leaves' capacity to assess the night length (from dusk to dawn). Therefore, the flowering process starts after a plant is exposed to a few successive nights longer than the critical day length. At this point, unifoliolate leaflets appear at stem node 1 (vegetative developmental phase V1 (leaves fully developed unifoliolate) and a young trifoliolate leaf appears at the second node. The induction continues after that in every consecutive leaf (Fehr and Caviness, 1977; Wilkerson et al. 1989).

Due to this short-day plant characteristic, the soybean genetic improvement process carried out over the years, especially in the $70 \mathrm{~s}$, through the manipulation of genes involved in flowering, using classical breeding methodologies, allowed its growth and development in several areas of the world and in different regions of producer's countries (Neumaier et al., 2000; Cai et al., 2020), either by delaying or advancing flowering, according to the geographical conditions (Cao et al., 2017; Cai et al., 2020). To be cultivated at higher latitudes, soybean breeders reduced the soybean sensitivity to the photoperiod, generating cultivars with a longer juvenile period (Farias et al., 2007; Lu et al., 2017).

Photoperiod sensitivity is genotype-dependent, and the response degree to the photoperiodic stimulus is a critical determinant of the adaptation region of a given crop. In sensitive soybean cultivars, the response to photoperiod is quantitative, which means that flowering will happen eventually, regardless of stimuli. Nevertheless, the period required for this will depend on the day length; on short-days, the induction is quicker than on long-days (Rodrigues et al., 2001). It is noteworthy that the too early flowering and early ripening of the soybean crop generally results in extremely low grain yields (Lu et al., 2017).

The inclusion of the long juvenile trait in soybean cultivars prolonged the vegetative phase and increased yield when grown under short-day conditions, which allowed the cultivation of the grain into the tropical regions and sowing seasons (Lu et al., 2017). Additionally, besides flowering time, the final yield in soybean is also related to plant architecture, including leaves, stem, branches, inflorescences, and pods in each node. So, to produce high-yield soybean varieties, coordination between the vertical growth, and branching is necessary (Pedersen and Lauer, 2004). For this reason, the manipulation of genes involved directly and indirectly in the flowering pathways has been used to increase the productivity and adaptability of the crop.

Despite all these advances, knowledge about the molecular bases of flowering in soybean is limited, as well as its relationship with genes involved in other mechanisms activated during this stage of development. Flowering triggers and pathways are dependent on the maturity genes in the plants and controlled by the plant hormones, developmental stage, temperature, and water availability, among other factors (Fornara et al., 2010; Wu and Hanzawa, 2014; Lyu et al., 2020; Ye et al., 2020). Physiologically, two main cyclic processes play an important role in the flowering mechanisms: 1) photoperiod (day length), that is the solar 24-hour cycle of day and night and, 2) a within-plant circadian rhythm.

Currently, available information indicates that floweringrelated genes in soybean can act as photoreceptors in response to blue (CRY1 - Cryptochrome Circadian Regulator 1, FKF1/2 - Flavin-binding, Kelch repeat, F-box 1 and 2, and ZTL3 Zeitlupe 3) and red (PHYB1 - Phytochrome B1 and PHYA1/2/3 - Phytochrome A1, A2, and A3) (Zhang et al., 2008; Wu et al., 2011; Xia et al., 2012; Xue et al., 2012) lights, acting as transcription factors (FT2a-Flowering Locus T 2a, FT5 a - Flowering Locus T 5a, FDL19 - Flowering Locus D9, LFY2 - Leafy 2, SOC1 - Suppressor of overexpression of CO1, APla - Apetala 1, SVP1 - Short vegetative phase 1, $F L C$-Flowering Locus C, and Ellb-E1 like-b protein), regulating the expression of downstream genes by binding to conserved cis-elements (Chi et al., 2011; Na et al., 2013; Su et al., 2013; Nan et al., 2014; Zhang et al., 2016; Zhu et al., 2018; Cai et al., 2020; Molinari et al., 2021); acting directly in the flowering process, such as VRN1 - Vernalization 1, ELF4 - Early Flowering 4, COL1a/b - Constans like 1a and $1 \mathrm{~b}$, and FT4 - Flowering Locus T 4 genes (Watanabe et al., 2011; Zhai et al., 2014; Cao et al., 2015; Suo et al., 2016; Marcolino-Gomes et al., 2017) and yet in epigenetic regulations (FLD - Flowering Locus D) (Hu et al., 2014).

To identify correlations between Arabidopsis thaliana and soybean, Jung et al. (2012) performed an analysis of gene orthology between species. After analyzing 183 genes of Arabidopsis thaliana with well-established roles in the flowering process, these authors identified 491 orthologs genes in soybean. This is explained by the two duplication events that the entire soybean genome underwent during its evolution, resulting in several copies of homologous genes, which were genes with only one copy in Arabidopsis (Schmutz et al., 2010).

Besides, another study crossed data from gene orthology with transcriptional analyzes in various soy tissues, including flowers and vegetables, under normal growing conditions. Results provided a set of genes involved in the flowering process that would possibly have similar responses in soybean (Libault et al., 2010; Severin et al., 2010).

Other studies had focused on the overexpression and/ or loss of gene function to access the function of these genes involved in the soybean flowering process (Table 1). Some authors inserted soybean genes in species such as Arabidopsis and tobacco, while others worked with soybean endogenous genes (cisgenesis). Table 1 summarizes the characteristics of these genes, such as phenotype related to flowering, their gene class, how their role was validated, and references. It is important to highlight that a common phenotype observed in the overexpression of a repressor gene is late flowering, while the overexpression of other genes (not repressors) resulted in early flowering. All these genes act together to regulate flowering, and some of these interactions can be seen in Figure 1. 


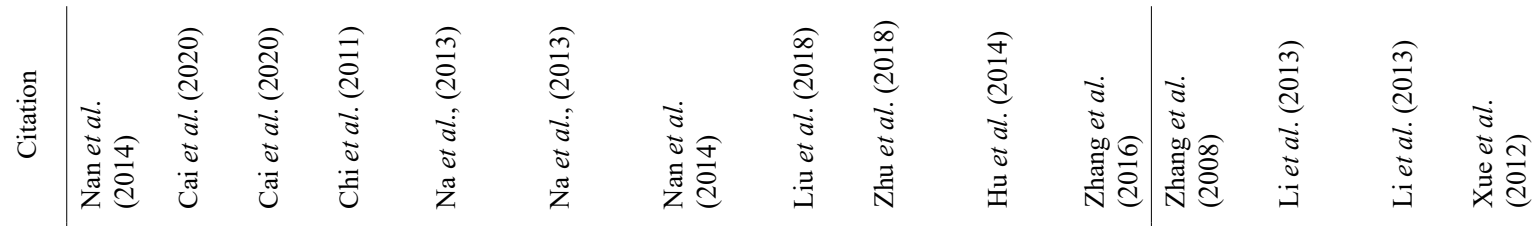

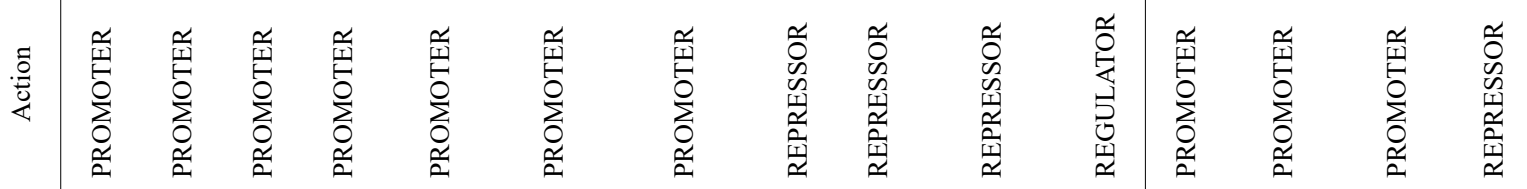

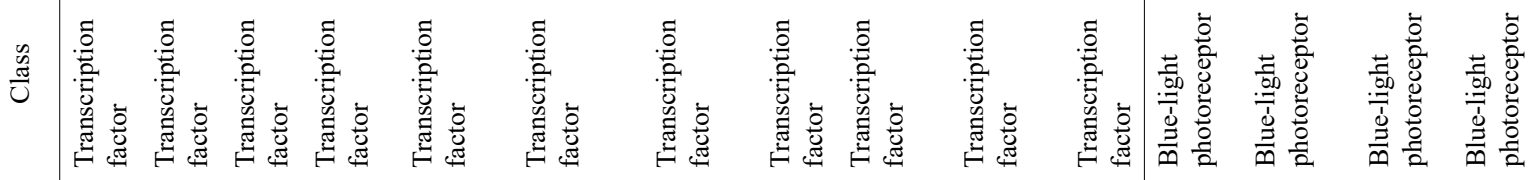

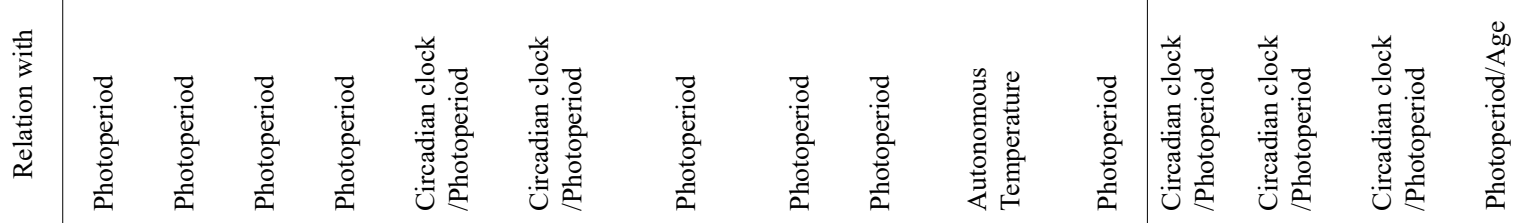

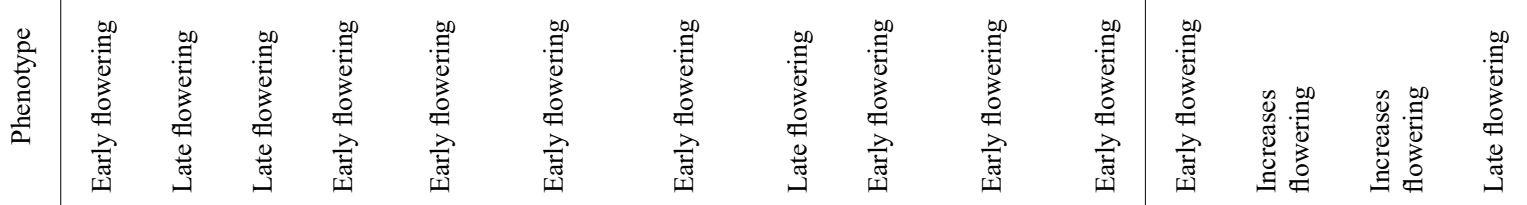

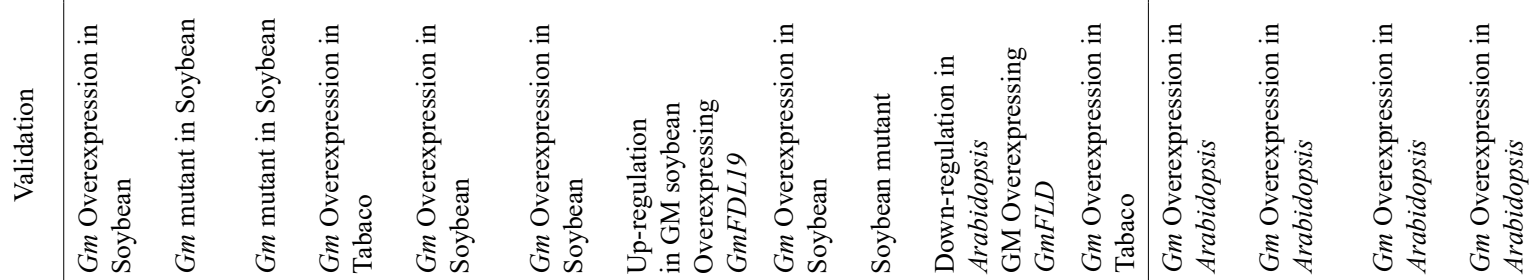



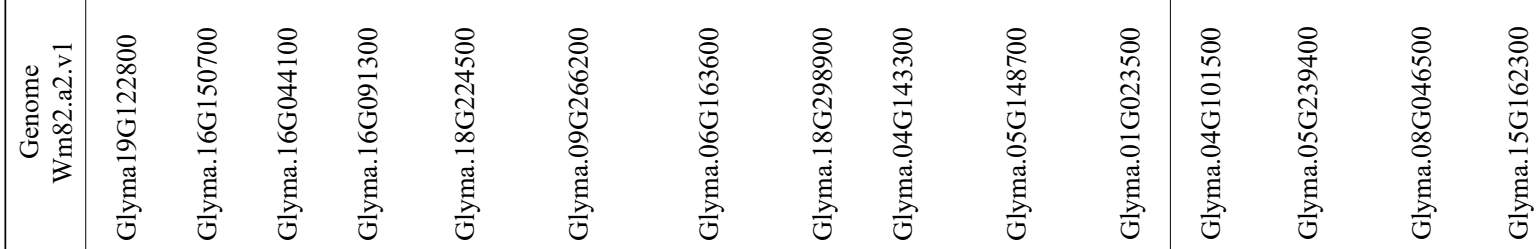




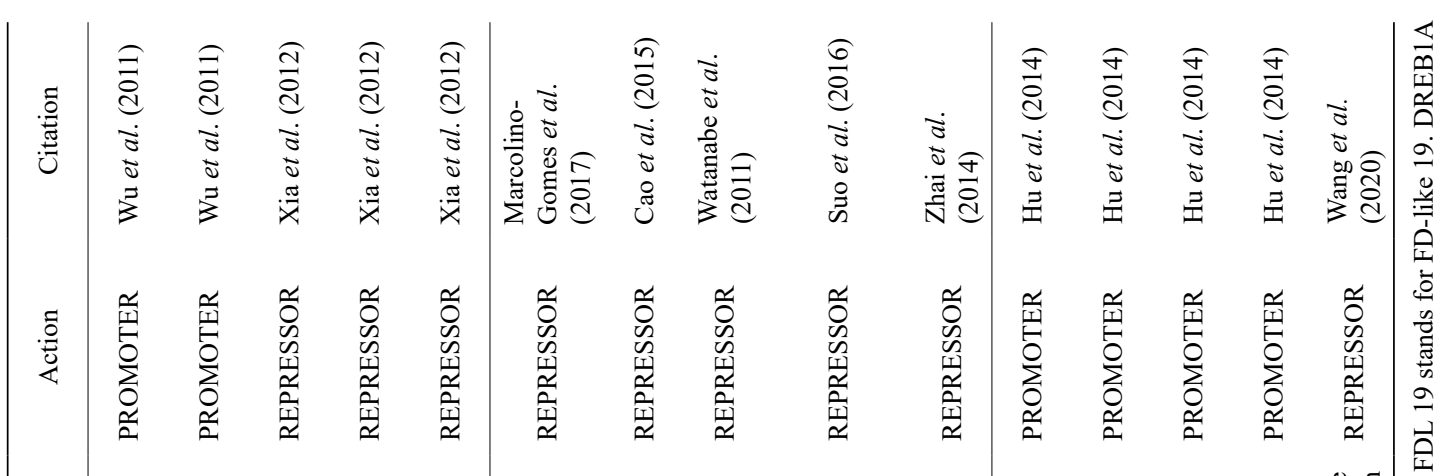

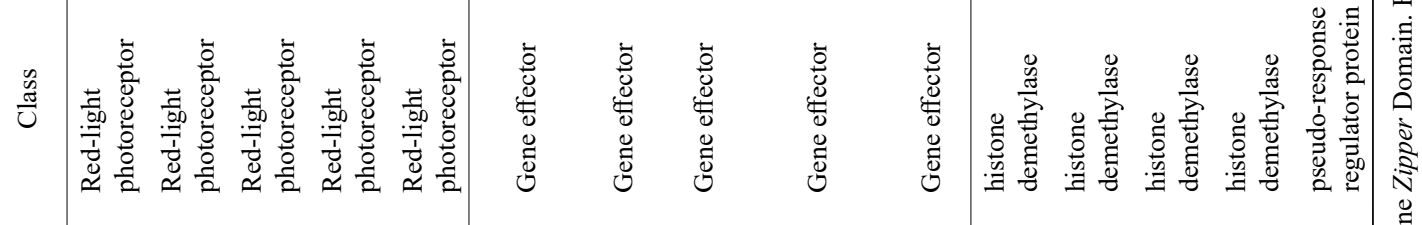

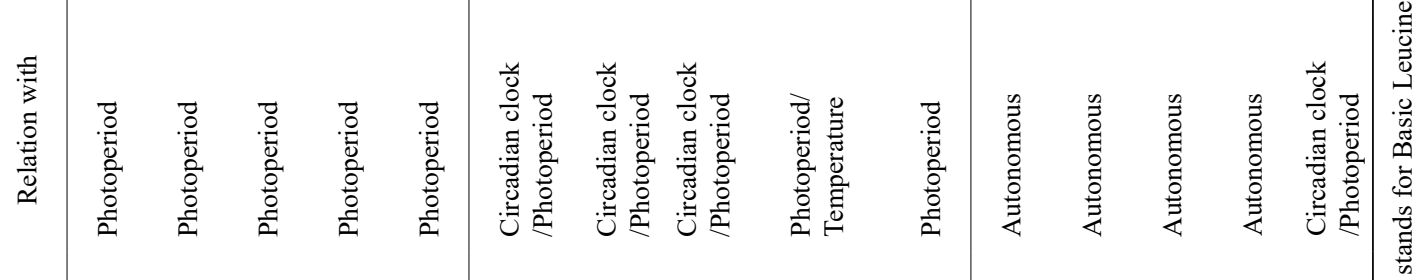

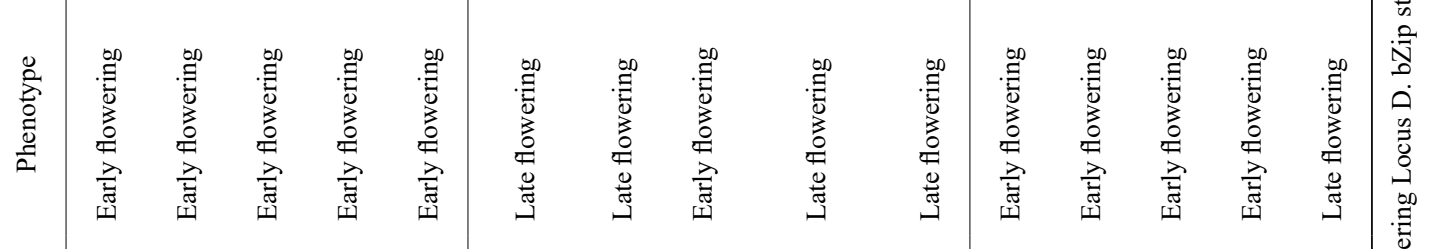

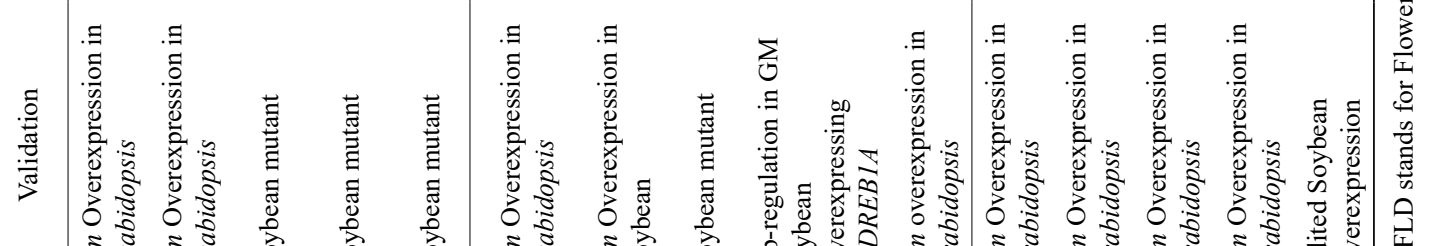

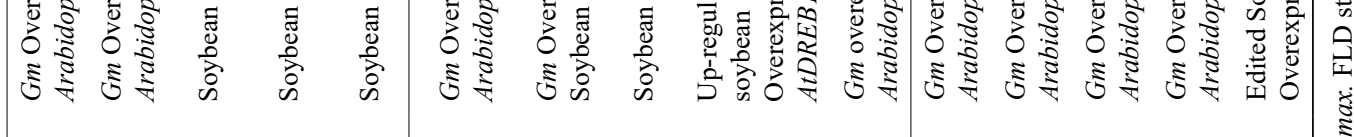

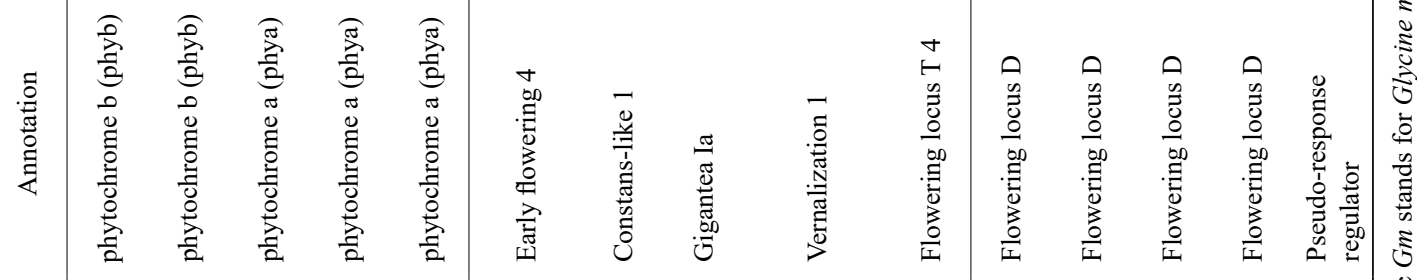

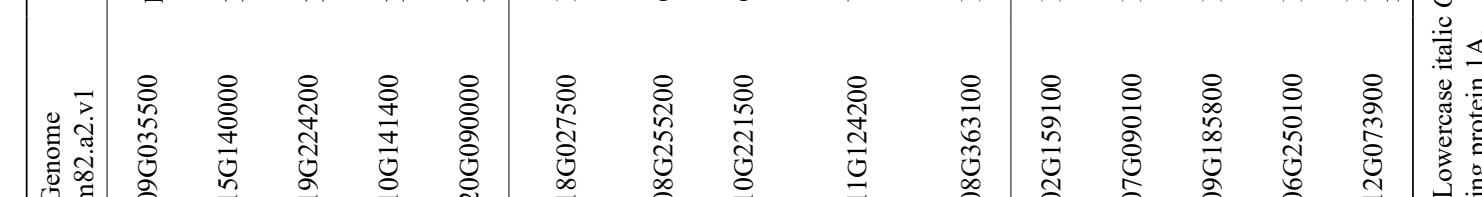

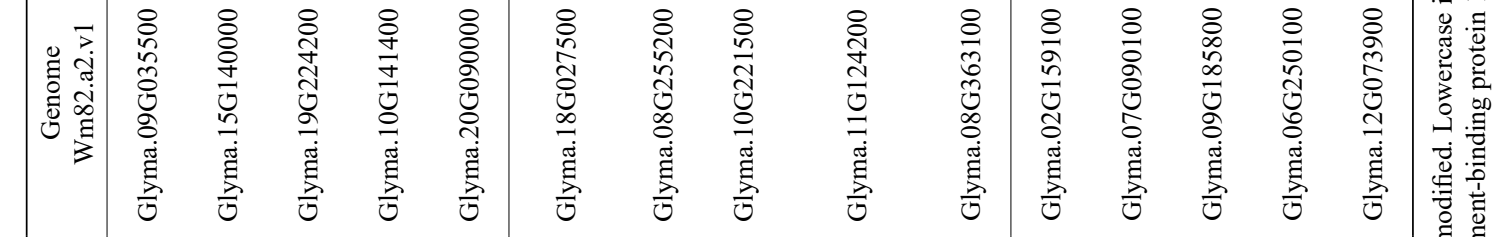

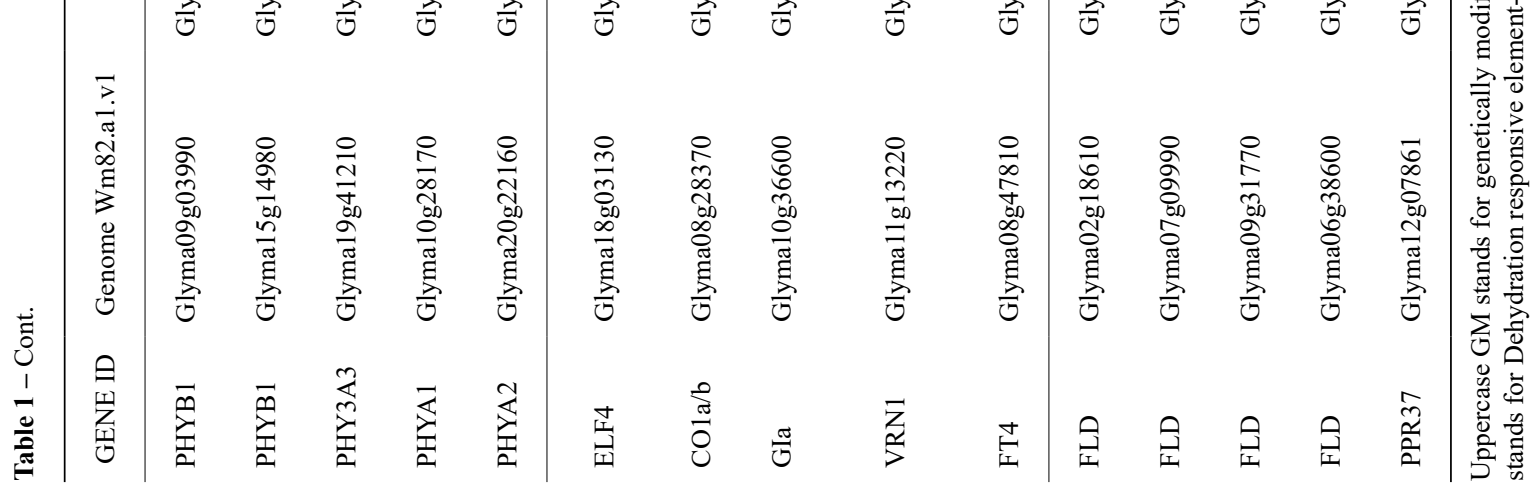




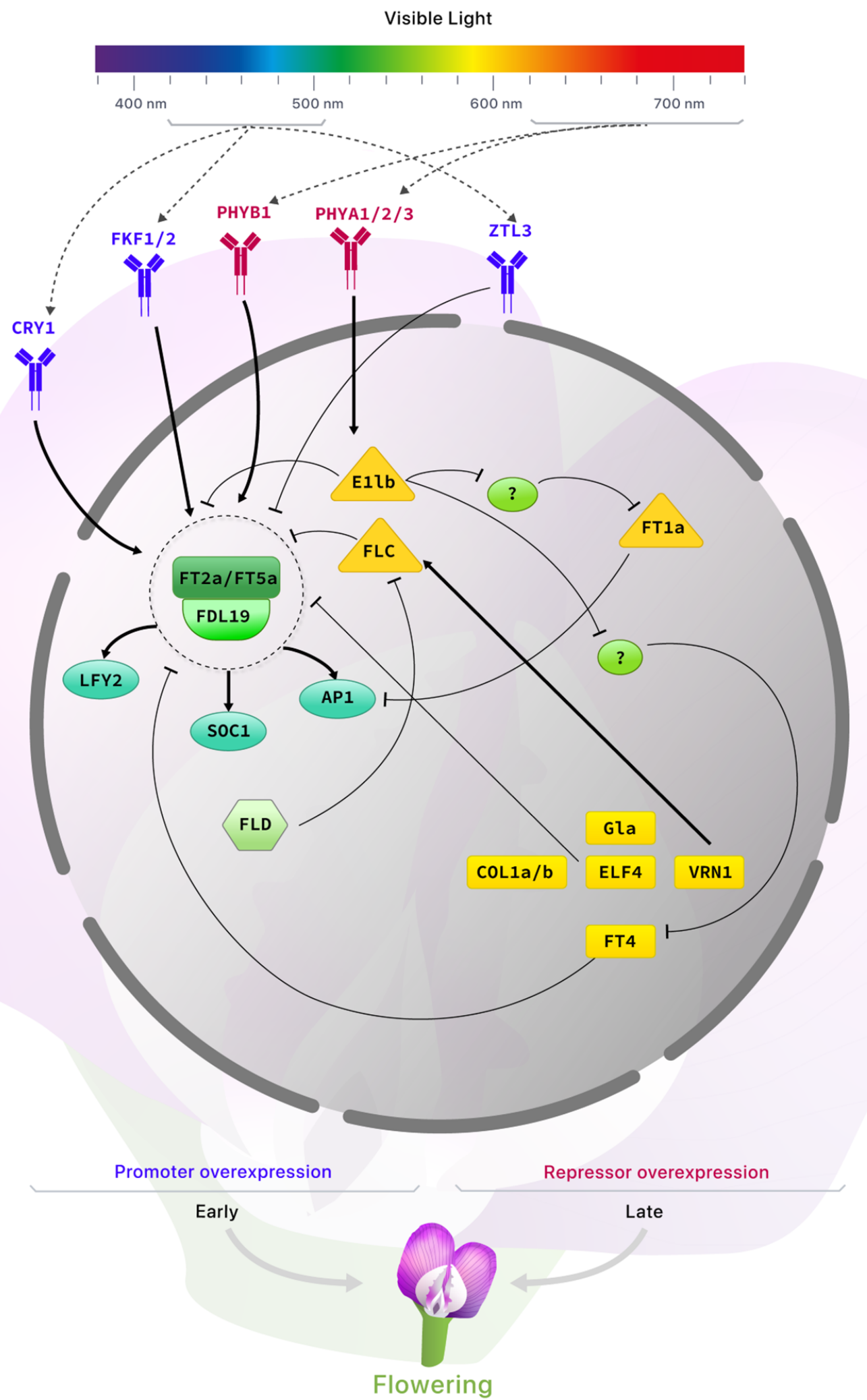

Figure 1 - Flowering controlling pathways in soybean. Straight end lines represent repression and arrows represent activation. Red: red-light photoreceptors. Blue: blue-light photoreceptors. Shades of green: flowering promoters. Shades of yellow: flowering repressors. Gray spaced circle represents the nucleus. Dotted small circle represents the central flowering complex. Dotted arrows represent the light spectrum absorbed by the photoreceptors. 
In general, plants start flowering after the vegetative developmental phase. During this process, called floral induction, the apical meristem of the shoot begins to produce flowers, not leaves. The metabolic trigger for flowering is controlled by a complex regulatory network that monitors the environmental changes, ensuring that floral induction happens under appropriate conditions, maximizing reproductive success through seed production (Fornara et al., 2010). Light is one of the principal environmental clues to flowering, but other climate factors such as drought can modify both floral anatomy and flowering time (Liscum et al., 2003; Fang et al., 2019; Luccioni et al., 2019).

Molecular studies in soybean have shown that the central regulating genes for flowering, GmFT2 $a$ and GmFT5a genes, are both flowering activators (Kong et al., 2010; Sun et al., 2011; Nan et al., 2014; Cai et al., 2020) and GmFTla (Flowering Locus T 1a) and GmFT4 genes, designated as flowering repressors (Zhai et al., 2014; Liu et al., 2018). These genes, regulated by the GmE1lb transcription factor (Zhai et al., 2014), are responsive to the photoperiod and the circadian cycle. Also, according to phylogenetic analyzes of its protein sequence, transcription factor $G m E 1 l b$ is specific to legumes (Xia et al., 2012) and its overexpression in soybean increased the expression levels of GmFT4 and GmFTla genes by suppressing unknown repressors of these genes (as shown in Figure 1). On the other hand, GmE1lb-overexpression repressed GmFT2a and GmFT5a expression (Zhai et al., 2014; Nan et al., 2014; Liu et al., 2018).

Flowering is a critical element of regional adaptability and geographic distribution of soybean, and it is strongly controlled by temperature and photoperiod. An analysis of quantitative trait locus (QTL) mapping indicated that the GmPRR37- Pseudo-response regulator 37 gene, which encodes a pseudo-response regulator protein, is accountable for the main QTL qFT12-2, identified in a population of 308 RILs (Recombinant Inbred Lines) (Wang et al., 2020). These lines resulting from a cross between an early-flowering cultivar, named Heihe27 (HH27) and the late-flowering soybean cultivar Zigongdongdou (ZGDD), assayed in many environments. Sequencing comparative analysis results confirmed that cultivar HH27 presented a non-sense mutation that occasioned the loss of the CCT domain in the GmPRR37 protein (Wang et al., 2020). Soybean Gmprr37- ZGDD mutants CRISPR/Cas9induced, growing under long-day (LD) conditions, showed early flowering (Wang et al., 2020). The overexpression of the GmPRR37 gene considerably delayed the flowering of GM soybean plants compared with WT, under conditions of long photoperiod. Furthermore, both the overexpression and the knockout of the GmPRR37 gene in soybean revealed no important phenotypic modifications in flowering time, under short- day (SD) conditions (Wang et al., 2020). Furthermore, under long-day (LD) situations, the expression of floweringpromoting FT homologs such as GmFT2 $a$ and GmFT5 $a$ was down-regulated by $G m P R R 37$ gene, while up-regulated the expression of flowering-inhibiting FT homolog GmFTla. Haplotype's analysis of the GmPRR37 gene in 180 cultivars harvested across China identified natural Gmprr37 mutants presenting earlier flowering, which allowed the cultivation of soybean at higher latitudes. This work revealed that the
GmPRR37 gene plays a key role in photoperiodic flowering and opens strategies to breed soybean cultivars adapted to specific farming systems and geographic regions (Wang et al., 2020).

Furthermore, studies have pointed out that the flowering repressing genes GmE1lb and GmFT4 showed suppression on short-days and, on the contrary, the stronger expression on long- days. Considering that the GmE1lb gene is soybean exclusive, the suggestion is that this crop developed a specific strategy to control the flowering time differing from the one observed in Arabidopsis thaliana. In this model plant, the overexpression of the GmFT4 gene delays flowering by 9 days (Zhai et al., 2014). In soybean, the GmFT4 gene may not be a direct target of $G m E 1 \mathrm{lb}$, as it acts as a transcription repressor. The GmFT4 gene is preferably induced over long-days, while the $G m F T 2 a / 5 a$ gene is preferably induced over short-days. GmFT4 and GmFT2a/5a genes oscillation throughout the day show an increase in expression at the beginning of dawn, a peak after $4 \mathrm{~h}$, a decrease at dusk, and then, a further increase, which suggests regulation by the circadian cycle (Zhai et al., 2014).

Besides genes already described, the GmFT1a acts as a floral repressor contributing to delay flowering time on soybean varieties, being a good candidate for genetic improvement to guarantee the successful implantation of high-yield germplasm in tropical environments. When analyzing the transcriptome of genetically modified soybean plants overexpressing the GmFTla gene, was observed that higher expression levels of GmFTla repress genes that specifying the identity of floral organs such as GmAPl (Liu et al., 2018). This gene in soybean can act downstream of GmFTla and contribute to the flowering transition (Nan et al., 2014). Interestingly, results obtained by Liu et al. (2018) with the overexpression of GmFTla were opposed to the regulatory pattern identified in a study performed by Nan et al. (2014). These authors observed that plants overexpressing flowering promoter genes such as GmFT2a and GmFT5 $a$ increased the levels of the GmAPl gene for specifying floral organ identity. These studies indicated that the flowering promoter gene $G m F T 2 a / 5 a$ and flowering inhibitor GmFTla gene can regulate the same set of genes in a competitive/antagonistic way that is still unclear (Liu et al., 2018).

Like GmEllb and GmFT4 genes, the GmFTla gene is induced in long-day conditions, inhibited in short-days, and when expressed, it acts delaying soybean flowering and maturation. GmFTla seems to be positively regulated by GmE1lb. Although GmFT1a acts as a transcription repressor, it may not be acting directly on $G m E 1 l b$, as observed in GmFT4 (Xia et al., 2012; Zhai et al., 2014; Liu et al., 2018). Liu et al. (2018) proposed that GmE1lb acts as a "switch", photoperiod-dependent, that positively regulates the expression of GmFTla flowering inhibitory genes, but negatively regulates the GmFT2 $a$ and GmFT5 $a$ flowering genes.

Nan et al. (2014) observed that the regulation of other flowering-related genes by floral activators, GmFT2a, and GmFT5a, occurs through their binding to the GmFDL19 gene. The formation of this complex (GmFT2a-GmFT5a$G m F D L 19$ ) will act on the positive regulation of genes such as $G m A P 1, G m S O C 1$, and $G m L F Y$. Thus, the suggestion is that the overexpression of this gene cascade promotes early 
flowering in soybean. Validation studies in tobacco have shown that GmAPl gene, when overexpressed, causes early flowering (Chi et al., 2011), as well as the overexpression of GmSOCl gene in soybean leads to early flowering as well (Na et al., 2013). It is noteworthy that both genes are considered activators of flowering.

Aiming to improve soybean yield, Cai et al. (2020) applied the CRISPR/Cas9 toolbox, to knock out the GmFT2a and the GmFT5 a flowering genes and obtain a double ft $2 a f t 5 a$ mutant. Under short-day conditions, the double $f t 2$ aft 5 a mutant bloomed about 31 days after the wild-type plants and preserved their vertical growth habit. During vegetative growth, these mutants produced significantly more nodes, leaf axis formed more branches, with an increase in the number of pods and seeds per plant, suggesting that the double ft 2 aft 5 a mutant has great potential to be introduced into the tropics.

Another known way of controlling the flowering induction in soybean occurs through the histone demethylase encoded by the GmFLD gene in the autonomous pathway. The GmFLD gene acts as a promoter of flowering by repressing the $A t F L C$ gene (through demethylation) and activating the expression of AtFT and AtSOCl in genetically modified Arabidopsis. The GmFLD gene suppresses the transcription of AtFLC through epigenetic mechanisms of histone modification close to the transcription site, decreasing the levels of $\mathrm{H} 3 \mathrm{~K} 4 \mathrm{me} 3$ (tri-methylation of the fourth lysine residue in histone H3) (Hu et al., 2014). In studies carried out by $\mathrm{Hu}$ et al. (2014), it was possible to observe that the overexpression of soybean gene GmFLD19 in an Arabidopsis fld mutant rescued the late-flowering phenotype.

Although the central point of flowering control involves transcription factors and flowering-related genes, as noted earlier, the onset of responses occurs through the perception of light by photoreceptors, which act upstream of the floral integrators GmFT2a/5a and GmFT4/1a. In Arabidopsis, both the overexpression of the endogenous blue-light photoreceptor $(A t Z T L)$ and its soybean analog (GmZTL3) generated delays in flowering in long-day conditions. Possibly, these genes have similar functions in soybean and Arabidopsis, as they are genes evolutionarily conserved, showing the same floweringrepression function (Xue et al., 2012). In Arabidopsis thaliana, the late flowering through overexpression of AtZTL occurs due to the strong reduction in transcriptional levels of AtFT (Kim et al., 2005).

While the overexpression of GmZTL3 causes late flowering, the overexpression of other blue light photoreceptors such as GmCRY1, GmFKF1, and GmFKF2 promotes earlier flowering in Arabidopsis thaliana (Zhang et al., 2008; Li et $a l ., 2013)$. These same authors observed that the activity of these blue light photoreceptors (flowering activators) occurs through the up-regulation of the AtFT gene. Arabidopsis plants overexpressing $G m C R Y 1$ showed an acceleration in flowering (Zhang et al., 2008), as well as overexpression of $G m F K F 1 / 2$ increased flowering in a short-day condition (Li et al., 2013).

Red light photoreceptors can also act as promoters $(P H Y B 1)$ and repressors (PHYBA1/2/3) of flowering. While $G m P H Y B 1$ acts by activating the $G m F T 2 a / 5 a$ gene, the GmPHYA gene acts by activating GmE1lb-like (Wu et al., 2011; Xia et al., 2012; Zhang et al., 2016). Another soybean gene involved in the flowering process, according to MarcolinoGomes et al. (2017), is GmELF4, responsive to the circadian cycle and the photoperiod. The overexpression of this gene in Arabidopsis thaliana delayed flowering when compared to wild-type plants. GmELF4 gene altered the expression of the AtFT gene negatively, explaining the observed floral delay (Marcolino-Gomes et al., 2017). Like GmELF4, the GmCOLIa/b, GmVRN1, and GmGIa - Gigantea genes were identified as repressors of flowering. According to Cao et al. (2015), the GmCOL1b mutant soybeans bloom earlier than WT plants, when growing in long-day conditions; and the overexpression of the GmCOLla gene delays flowering. These authors also observed that the overexpression of the GmCOL1a gene led to the downregulation of GmFT2a and GmFT5 $a$ genes, illustrating the activity of GmCOL 1a gene as a flowering suppressor in soybean. In another study carried out by Watanabe et al. (2011), it was observed that the soybean $e 2 / e 2$ genotype (mutant for GmGIa) showed early flowering, inducing the expression of the GmFT2a gene, and showing the role of $G I$ as a flowering repressor.

Still, in a study performed by Suo et al. (2016), the relationship of a gene involved in flowering ( $G m V R N 1$ like) with a drought-tolerance gene (AtDREBIA Dehydration-responsive element binding 1A) was reported. The overexpression of DREB1A genes resulted in drought tolerance, and also caused delayed flowering (Kidokoro et al., 2015; Zhou et al., 2020). According to these authors, in genetically modified soy plants overexpressing this gene, the GmVRN1 gene, homologous to Arabidopsis, was strongly induced. This fact was explained by the link of this transcription factor to DRE motifs (ACCGAC) in the promoter region of the GmVRN1 gene, suggesting that the late flowering of genetically modified plants occurred due to the positive regulation of VRN1 in soybean, which is considered a flowering repressor. Previously, in another study conducted by the same authors, the overexpression of DREB genes caused the flowering delay by activating the floral repressor AtFLC in Arabidopsis (Seo et al., 2009 apud Suo et al., 2016).

The SVP genes can also present different actions on the flowering time among species. According to Fornara et al. (2010), the AtSVP gene, a flowering repressor in Arabidopsis, can be associate with the AtFLC gene and inactivate the AtFT gene. The overexpression of the MtSVP gene from Medicago, for example, caused a delay in flowering in Arabidopsis thaliana (Jaudal et al., 2013), as well as the cisgenic overexpression of AtSVP (Hartmann et al., 2000); while the overexpression of soybean GmSVP1 gene in tobacco accelerates flowering time (Zhang et al., 2016). These findings demonstrated the importance of studies on species-specific gene manipulation since the same gene can present different responses depending on the species in which it was inserted. Although the role of the SVP gene in soybean is not yet fully understood, in Arabidopsis, a study carried out by Wang et al. (2018) showed the correlation of this gene with drought. These authors showed that the overexpression of the AtSVP gene confers drought tolerance in Arabidopsis thaliana by regulating ABA catabolism.

All these reports show that the manipulation of genes to delay/accelerating the flowering process has been widely 
used to increase productivity, either by the overexpression of repressors or by mutation of flowering promoters. As other examples, the overexpression of the AtFLC gene, a flowering repressor, in tobacco, delayed flowering by 36 days and significantly increased biomass production when compared to wild-type plants (Salehi et al., 2005). Additionally, the mutation of flowering promoter genes such as GmFT2 $a$ and GmFT5a, in soybean, delayed grain flowering by 31 days and produced many more pods resulting in a substantial increase in the number of seeds (Cai et al., 2020).

\section{Flowering time and its relationship with drought- response}

Flowering is not only an essential part of the plant's reproductive process but also a critical developmental stage vulnerable to environmental stresses such as drought (Kazan and Lyons, 2016). Exposure to water deficit during this period can cause substantial yield losses in seed-producing plants. However, it is becoming increasingly evident that altering flowering time is an evolutionary strategy adopted by plants to maximize the chances of reproduction under diverse stress conditions, ranging from pathogen infection to heat, salinity, and drought. A better understanding of how complex environmental variables affect plant phenology is important for future genetic manipulation of crops aiming to increase productivity under a changing climate (Kazan and Lyons 2016; Molinari et al., 2021). The manipulation of different flowering genes triggered modifications in flowering time and drought adaptability. Publications showing this possibility were summarized in Table 2 . The overexpression of flowering promoters for example triggers the development of plants more tolerant to drought and, the mutation of flowering repressors could give the plant the capacity to escape drought, by flowering earlier. Drought escape is an adaptive mechanism that enables plants to complete their life cycle before a drought event. Early flowering time and a shorter vegetative phase can be very important for production in conditions of terminal drought, since it can minimize exposure to dehydration during flowering and, consequently, losses in grain filling and final yield (Shavrukov et al., 2017; Molinari et al., 2021).

Most flowering genes have had their functions in response to drought validated in Arabidopsis thaliana. Due to the similarity of the genes involved in the flowering process, it is possible to infer that the manipulation of soybean flowering genes can also result in the same response to drought. Among all the mentioned genes, only $S P V$ has antagonistic functions among species. In Arabidopsis is a flowering repressor, and in soybean is a flowering promoter (Fornara et al., 2010; Zhang et al., 2016).

Amongst genes with the same function, in Arabidopsis, the flowering promoters $P H Y B$ and $S O C 1$, when overexpressed, generated plants more tolerant to drought through earlier flowering process (González et al., 2012; Hwang et al., 2019). The overexpression of flowering promoter $P H Y B$ enhanced drought tolerance in adult plants of Arabidopsis thaliana by increasing stomatal sensitivity to ABA, when water becomes a scarce resource (González et al., 2012). The mechanism underlying $P H Y B$-enhanced drought tolerance could be the result of a higher capacity of wild-type plants to extract soil water, in comparison to $p h y b$ mutant. This osmotic adjustment is a typical response to drought, which can improve the chances of acquiring water from drying soil (González et al., 2012).

Similarly, according to Hwang et al. (2019), in Arabidopsis thaliana soc1 mutant plants, a reduction in drought-escape response was observed. This data suggests that the overexpression of SOC1 might contribute to adaptation by enabling plants to complete their life cycles under drought.

In soybean, the overexpression of the flowering promoter GmFLD19 also generated plants more tolerant to water deficit, by shortening their life cycle. This gene is a transcription factor that belongs to the basic leucine zipper (bZIP) family. $b Z I P$ genes play an important role in the growth and developmental process, as well as response to various abiotic stresses, such as drought and, high salinity (Li et al., 2017). Besides that, the overexpressing of GmFDL19 also causes early flowering in transgenic soybean plants (Hu et al., 2014; Li et al., 2017). GmFDL19 likewise enhanced tolerance to drought and, salt stress in soybean and, it is highly induced by abscisic acid (ABA), an important phytohormone, and polyethylene glycol (PEG 6000) (Li et al., 2017). The overexpression of GmFDL19 in soybean enhanced drought and salt tolerances at the seedling stage. Moreover, the relative plant height and the relative shoot dry weight of transgenic plants were significantly higher than those of the WT, under drought. Furthermore, GmFDL19 expression reduced the accumulation of $\mathrm{Na}+$ ion content, up-regulated the expression of several $\mathrm{ABA} /$ stress-responsive genes in transgenic soybean and increased the activities of several antioxidative enzymes and chlorophyll content, but reduced malondialdehyde content (Li et al., 2017). According to these authors, GmFDL19 has potential to improve multiple stress tolerance in transgenic soybean lines.

Table 2 - Flowering genes in soybean and its relationship with drought-response.

\begin{tabular}{|c|c|c|c|c|c|c|}
\hline GENE ID & Validation & Specie & Action & $\begin{array}{l}\text { Flowering } \\
\text { expected }\end{array}$ & Drought mechanism & Citation \\
\hline FDL19 & Overexpression & Soybean & PROMOTER & Early & Escape & Li et al., 2017 \\
\hline SOC1 & Overexpression & Arabidopsis thaliana & PROMOTER & Early & Escape & Hwang et al., 2019 \\
\hline РНYB & Overexpression & Arabidopsis thaliana & PROMOTER & Early & Escape & Gonzalez et al., 2012 \\
\hline ELF4 & Mutation & Soybean/Arabidopsis & REPRESSOR & Early & Escape & Jin et al., 2020 \\
\hline CRY1 & Mutation & Arabidopsis thaliana & PROMOTER & Late & Tolerance & Mao et al., 2005 \\
\hline GIa & Overexpression & Arabidopsis thaliana & REPRESSOR & Late & Tolerance & Baek et al., 2020 \\
\hline
\end{tabular}


In contrast, the mutation of flowering promoter $C R Y 1$ confers enhanced drought tolerance in Arabidopsis thaliana, and the overexpression of $C R Y 1$ resulted in greater water loss (Mao et al., 2005). This involvement of Arabidopsis CRY1 promoters in drought response is largely dependent on their functions in inducing stomatal opening in response to blue light (Mao et al., 2005). In soybean, it has been shown that cryptochromes GmCRYla affect blue light inhibition of cell elongation. In soybean, GmCRYla seems to be the more predominant regulator of photoperiodic flowering. The photoperiod-dependent circadian rhythmic expression of the GmCRYla protein correlated with photoperiodic flowering and latitudinal distribution of different soybean accessions (Zhang et al., 2008).

Studies involving flowering repressors showed that the inhibition of ELF4 in Arabidopsis thaliana and soybean, advances the flowering period. Besides that, it was observed that the inhibition of the flowering repressor GmELF4, increased drought tolerance in soybean. This gene could be manipulated to breed drought-tolerant varieties (Jin et al., 2020). In corroboration, when the ELF4 gene was overexpressed the soybean plants, they became sensitive to drought (Jin et al., 2020).

Lastly, GI, a key regulator of photoperiod-dependent flowering and the circadian rhythm, is also involved in the signaling pathways for various abiotic stresses, like drought. The Arabidopsis thaliana gil mutants are hypersensitive to drought due to the uncontrolled water loss triggered by the reduction of abscisic acid levels. This data suggested that the GI positively regulates diurnal ABA synthesis by affecting the expression of NCED3 - 9-cis-epoxycarotenoid dioxygenase 3 gene, contributing to drought tolerance (Baek et al., 2020; Molinari et al., 2020). For this reason, during drought stress, GI transcription is up-regulated (Han et al., 2013). These results give evidence that the overexpression of GI could potentially increase drought tolerance.

Finally, as a complement to all these studies, an improvement of knowledge about soybean flowering interaction pathways involved in the responses to drought, through the identification of genes differentially expressed in soybean flowers and pods under this stress condition would give more information and, new insights into the research focused on developing more drought-tolerant soybean lines, with fewer losses, as a result of flowers or pods abortion, and consequently productivity.

\section{Acknowledgments}

We would like to thank the National Council for Scientific and Technological Development (CNPq) and the Coordination for the Improvement of Higher Education Personnel (CAPES) for granting scholarships for some of the authors. This research was funded by Empresa Brasileira de Pesquisa Agropecuária (Embrapa), INCT BioSyn (National Institute of Science and Technology in Synthetic Biology), CNPq (National Council for Scientific and Technological Development), and FAPDF (Research Support Foundation of the Federal District), Brazil.

\section{Conflict of interest}

The authors declare that there is no conflict of interest that could be perceived as prejudicial to the impartiality of the reported research.

\section{Author Contributions}

MDCM conceptualization, writing-original draft, visualization - first writing; RFP conceptualization, writing - review \& editing, visualization - first writing; SRRM and DAB writing - review \& editing; DRM writing - review \& editing; ELR supervision, writing - review \& editing; LMMH supervision, writing - review \& editing; ALN supervision, writing - review \& editing.

\section{References}

Baek D, Kim WY, Cha JY, Park HJ, Shin G, Park J and Yun DJ (2020) The GIGANTEA-ENHANCED EM LEVEL complex enhances drought tolerance via regulation of abscisic acid synthesis. Plant Physiol 184:443-458.

Cai Y, Wang L, Chen L, Wu T, Liu L, Sun S, Wu C, Yao W, Jiang B, Yuan S et al. (2020) Mutagenesis of GmFT2a and GmFT5a mediated by CRISPR/Cas9 contributes for expanding the regional adaptability of soybean. Plant Biotechnol J 18: 298-309.

Cao D, Li Y, Lu S, Wang J, Nan H, Li X, Shi D, Fang C, Zhai H, Yuan X et al. (2015) GmCOL1a and GmCOL1b function as flowering repressors in soybean under long-day conditions. Plant Cell Physiol 56:2409-2422.

Cao D, Takeshima R, Zhao C, Liu B, Jun A and Kong F (2017). Molecular mechanisms of flowering under long days and stem growth habit in soybean. J Exp Bot 68:1873-1884.

Chi Y, Huang F, Liu H, Yang S and Yu D (2011) An APETALA1-like gene of soybean regulates flowering time and specifies floral organs. J. Plant Physiol 168:2251-2259.

Desclaux D, Huynh T-T and Roumet P (2000) Identification of soybean plant characteristics that indicate the timing of drought stress. Crop Sci 40:716-722.

Fang C, Chen L, Nan H, Kong L, Li Y, Zhang H, Dong L, Li H, Li T, Tang Y et al. (2019) Rapid identification of consistent novel QTLs underlying long-juvenile trait in soybean by multiple genetic populations and genotyping-by-sequencing. Mol Breed 39:80.

Farias JRB, Nepomuceno AL and Neumaier N (2007) Ecofisiologia da soja. Embrapa Soja, Londrina, 8 p.

Fehr WR and Caviness CE (1977) Stages of soybean development. Iowa State University, Iowa, Special Report 87.

Ferreira RC (2016) Quantificação das perdas por seca na cultura da soja no Brasil. Doctoral Thesis, Universidade Estadual de Londrina, Londrina, $99 \mathrm{p}$.

Fornara F, Montaigu A and Coupland G (2010) SnapShot: Control of flowering in Arabidopsis. Cell 141:550-550. e5502.

González CV, Ibarra SE, Piccoli PN, Botto JF and Boccalandro HE (2012) Phytochrome B increases drought tolerance by enhancing ABA sensitivity in Arabidopsis thaliana. Plant Cell Environ 35:1958-1968.

Han Y, Zhang X, Wang Y and Ming F (2013) The suppression of WRKY44 by GIGANTEA-miR172 pathway is involved in drought response of Arabidopsis thaliana. PLoS One 8:e73541.

Hartmann U, Höhmann S, Nettesheim K, Wisman E, Saedler H and Huijser P (2000) Molecular cloning of SVP: A negative regulator of the floral transition in Arabidopsis. Plant J 21:351-360. 
Hu Q, Jin Y, Shi H and Yang W (2014) GmFLD, a soybean homolog of the autonomous pathway gene FLOWERING LOCUS D, promotes flowering in Arabidopsis thaliana. BMC Plant Biol 14:263.

Hwang K, Susila H, Nasim Z, Jung JY and Ahn JH (2019) Arabidopsis $\mathrm{ABF} 3$ and $\mathrm{ABF} 4$ transcription factors act with the NF-YC complex to regulate SOC1 expression and mediate droughtaccelerated flowering. Mol Plant 12:489-505.

Jaudal M, Monash J, Zhang L, Wen J, Mysore KS, Macknight R and Putterill J (2013) Overexpression of Medicago SVP genes causes floral defects and delayed flowering in Arabidopsis but only affects floral development in Medicago. J Exp Bot 65:429-442

Jin Y, Zhao H, Lamboro A, Xiao Z, Zhang Q, Guan S and Wang P (2020) Cloning and functional verification of gene GmELF4LIKE4 in soybean [Glycine max (L.) Merr] BMC Biotechnol preprint 1-14.

Jung CH, Wong CE, Singh MB and Bhalla PL (2012) Comparative genomic analysis of soybean flowering genes. PLoS One $7: e 38250$.

Kazan K and Lyons R (2016) The link between flowering time and stress tolerance. J Exp Bot. 67:47-60.

Kidokoro S, Watanabe K, Ohori T, Moriwaki T, Maruyama K, Mizoi J, Htwe NMPS, Fujita Y, Sekita S and Shinozaki K (2015) Soybean DREB 1/CBF-type transcription factors function in heat and drought as well as cold stress-responsive gene expression. Plant J 81:505-518.

Kim W-Y, Hicks KA and Somers DE (2005) Independent roles for EARLY FLOWERING 3 and ZEITLUPE in the control of circadian timing, hypocotyl length, and flowering time. Plant Physiol 139:1557-1569.

Kong F, Liu B, Xia Z, Sato S, Kim BM, Watanabe S and Abe J (2010) Two coordinately regulated homologs of FLOWERING LOCUS T are involved in the control of photoperiodic flowering in soybean. Plant Physiol 154:1220-1231.

Li F, Zhang X, Hu R, Wu F., Ma J, Meng Y and Fu Y (2013) Identification and molecular characterization of FKF1 and GI homologous genes in soybean. PLoS One 8:e79036.

Li Y, Chen Q, Nan H, Li X, Lu S, Zhao X and Cao D (2017). Overexpression of GmFDL19 enhances tolerance to drought and salt stresses in soybean. PLoS One 12:e0179554.

Libault M, Farmer A, Joshi T, Takahashi K, Langley RJ, Franklin LD and Stacey G (2010) An integrated transcriptome atlas of the crop model Glycine max, and its use in comparative analyses in plants. Plant J 63:86-99.

Licht MA, Wright D and Lenssen AW (2013) Soybean response to drought. Iowa State University, Iowa.

Liscum E, Hodgson DW and Campbell TJ (2003) Blue light signaling through the cryptochromes and phototropins. So that's what the blues is all about. Plant Physiol 133:1429-1436.

Liu W, Jiang B, Ma L, Zhang S, Zhai H, Xu X, Hou W, Xia Z, Wu C, Sun S et al. (2018) Functional diversification of Flowering Locus Thomologs in soybean: GmFT1a and GmFT2a/5a have opposite roles in controlling flowering and maturation. New Phytol 217:1335-1345.

Lu S, Zhao X, Hu Y, Liu S, Nan H, Li X, Fang C, Cao D, Shi X, Kong L et al. (2017) Natural variation at the soybean J locus improves adaptation to the tropics and enhances yield. Nat Genet 49:773-779.

Luccioni L, Krzymuski M, Sánchez-Lamas M, Karayekov E, Cerdán PD and Casal JJ (2019) Constans delays Arabidopsis flowering under short days. Plant J 97:923-932.

Lyu J, Cai Z, Li, Y Suo H, Yi R, Zhang S and Nian H (2020) The floral repressor GmFLC-like is involved in regulating flowering time mediated by low temperature in soybean. Int J Mol Sci 21:1322.
Mao J, Zhang YC, Sang Y, Li QH and Yang HQ (2005) A role for Arabidopsis cryptochromes and COP1 in the regulation of stomatal opening. Proc Natl Acad Sci U S A 102:12270-12275.

Marcolino-Gomes J, Nakayama TJ, Molinari HBC, Basso MF, Henning LMM, Fuganti-Pagliarini R, Harmon FG and Nepomuceno AL (2017) Functional characterization of a putative Glycine max ELF4 in transgenic Arabidopsis and its role during flowering control. Front Plant Sci 8:618.

Molinari MDC, Fuganti-Pagliarini R, Marcolino-Gomes J, Barbosa DA, Marin SRR, Mertz-Henning LM and Rech Filho EL (2021). Flower and pod genes involved in soybean sensitivity to drought. J Plant Int 16:187-200.

Molinari MDC, Fuganti-Pagliarini R, Marin SRR, Ferreira LC, Barbosa DA, Marcolino-Gomes $\mathrm{J}$ and Nepomuceno AL (2020) Overexpression of AtNCED3 gene improved drought tolerance in soybean in greenhouse and field conditions. Genet Mol Biol 43:e20190292.

Na X, Jian B, Yao W, Wu C, Hou W, Jiang B, Bi Y and Han T (2013) Cloning and functional analysis of the flowering gene GmSOC1-like, a putative suppressor of overexpression CO1/ Agamous-like 20 (SOC1/AGL20) ortholog in soybean. Plant Cell Rep 32:1219-1229.

Nan H, Cao D, Zhang D, Li Y, Lu S, Tang L, Yuan X, Liu B and Kong F (2014) GmFT2a and GmFT5a redundantly and differentially regulate flowering through interaction with and upregulation of the bZIP transcription factor GmFDL19 in soybean. PLoS One 9:e97669.

Neumaier N, Nepomuceno AL, Farias JRB and Oya T (2000) Estádios de desenvolvimento da cultura de soja. Embrapa Trigo, Passo Fundo, 9 p.

Pedersen P and Lauer JG (2004) Response of soybean yield components to management system and planting date. Agron J 96:1372-1381.

Rodrigues O, Didonet AD, Lhamby JCB, Bertagnolli PF and Luz JS (2001) Resposta quantitativa do florescimento da soja à temperatura e ao fotoperíodo. Pesqui Agropecu Bras 36:431437.

Salehi H, Ransom CB, Oraby HF, Seddighi Z and Sticklen MB (2005) Delay in flowering and increase in biomass of transgenic tobacco expressing the Arabidopsis floral repressor gene FLOWERING LOCUS C. J Plant Physiol 162:711-717.

Schmutz J, Cannon SB, Schlueter J, Ma J, Mitros T, Nelson W and Xu D (2010) Genome sequence of the palaeopolyploid soybean. Nature 463:178-183.

Seo E, Lee H, Jeon J, Park H, Kim J, Noh YS and Lee I (2009). Crosstalk between cold response and flowering in Arabidopsis is mediated through the flowering-time gene SOC1 and its upstream negative regulator FLC. Plant Cell 21:3185-3197.

Severin AJ, Woody JL, Bolon YT, Joseph B, Diers BW, Farmer AD, Muehlbauer GJ, Nelson RT, Grant D, Specht JE et al. (2010) RNA-Seq Atlas of Glycine max: A guide to the soybean transcriptome. BMC Plant Biol 10:160.

Shavrukov Y, Kurishbayev A, Jatayev S, Shvidchenko V, Zotova L, Koekemoer F and Langridge P (2017) Early flowering as a drought escape mechanism in plants: How can it aid wheat production? Front plant sci 8:1950.

Su Z, Ma X, Guo H, Sukiran NL, Guo B, Assmann SM and Ma H (2013). Flower development under drought stress: Morphological and transcriptomic analyses reveal acute responses and long-term acclimation in Arabidopsis. Plant Cell 25:3785-3807.

Sun H, Jia Z, Cao D, Jiang B, Wu C, Hou W, Liu Y, Fei Z, Zhao D and Han T (2011) GmFT2a, a soybean homolog of FLOWERING LOCUS T, is involved in flowering transition and maintenance. PLoS One 6:e29238. 
Suo H, Lü J, Ma Q, Yang C, Zhang X, Meng X, Huang S and Nian $H$ (2016) The AtDREB1A transcription factor up-regulates expression of a vernalization pathway gene, GmVRN1-like, delaying flowering in soybean. Acta Physiol Plant 38:137.

Wang L, Sun S, Wu T, Liu L, Sun X, Cau, Y, Li J, Jia H, Yuan S et al. (2020) Natural variation and CRISPR/Cas9-mediated mutation in GmPRR37 affect photoperiodic flowering and contribute to regional adaptation of soybean. Plant Biotechnol J 18:1184-1197.

Wang Z, Wang F, Hong Y, Yao J, Ren Z, Shi, H and Zhu JK (2018) The flowering repressor SVP confers drought resistance in Arabidopsis by regulating abscisic acid catabolism. Mol Plant 11:1184-1197.

Watanabe S, Xia Z, Hideshima R, Tsubokura Y, Sato S, Yamanaka N, Takahashi R, Anai T, Tabata S, Kitamura K et al. (2011) A map-based cloning strategy employing a residual heterozygous line reveals that the GIGANTEA gene is involved in soybean maturity and flowering. Genetics 188:395-407.

Wilkerson GG, Jones JW, Boote KJ and Buol GS (1989) Photoperiodically sensitive interval in time to flower of soybean. Crop Sci 29:721-726.

Wu F and Hanzawa Y (2014) Photoperiodic control of flowering in plants. In: Pessarakli M (ed) Handbook of Plant and Crop Physiology. CRC Press, New York, pp 150-167.

Wu FQ, Zhang XM, Li DM and Fu YF (2011) Ectopic expression reveals a conserved PHYB homolog in soybean. PLoS One 6:e27737.

Xia Z, Watanabe S, Yamada T, Tsubokura Y, Nakashima H, Zhai H, Anai T, Sato S, Yamazaki T, Lü S et al. (2012) Positional cloning and characterization reveal the molecular basis for soybean maturity locus E1 that regulates photoperiodic flowering. Proc Natl Acad Sci U S A 109:E2155-E2164.

Xue ZG, Zhang XM, Lei CF, Chen, XJ and Fu YF (2012) Molecular cloning and functional analysis of one ZEITLUPE homolog GmZTL3 in soybean. Mol Biol Rep 39:1411-1418.

Ye H, Ren F, Guo H, Guo L, Bai J and Wang Y (2020) Identification of key genes and transcription factors in ageing Arabidopsis papilla cells by transcriptome analysis. Plant Physiol Biochem 147:1-9.
Zhai H, Lü S, Liang S, Wu H, Zhang X, Liu B, Kong F, Yuan X, Li J and Xia Z (2014) GmFT4, a homolog of FLOWERING LOCUS T, is positively regulated by $\mathrm{E} 1$ and functions as a flowering repressor in soybean. PLoS One 9:e89030.

Zhang H, Yan H, Zhang D and Yu D (2016). Ectopic expression of a soybean SVP-like gene in tobacco causes abnormal floral organs and shortens the vegetative phase. Plant Growth Regul 80:345-353.

Zhang Q, Li H, Li R, Hu R, Fan C, Chen F and Lin C (2008) Association of the circadian rhythmic expression of GmCRY 1a with a latitudinal cline in photoperiodic flowering of soybean. Proc Natl Acad Sci U S A 105:21028-21033.

Zhou Y, Chen M, Guo J, Wang Y, Min D, Jiang Q, Hutai J, Huang C, Wei W and Xu Het al. (2020) Overexpression of the soybean (Glycine max) DRE-binding transcription factor GmDREB1 enhanced drought stress tolerance of transgenic wheat in the field. J Exp Bot 71:1842-1857.

Zhu J, Takeshima R, Harigai K, Xu M, Kong F, Liu B, Kanazawa A, Yamada T and Abe J (2018) Loss of function of the E1Like-b gene associates with early flowering under long-day conditions in soybean. Front Plant Sci 9:1867.

\section{Internet Resources}

Companhia Nacional de Abastecimento (CONAB) Grãos por produto, https://www.conab.gov.br/info-agro/safras/serie-historica-dassafras (August 15, 2021).

Intergovernmental Panel on Climate Change (IPCC) (2021) Climate change widespread, rapid, and intensifying - IPCC, https:// www.ipcc.ch/site/assets/uploads/2021/08/IPCC_WGI-AR6Press-Release_en.pdf (August 15, 2021).

United States Department of Agriculture (USDA) Crop Explorer - Production Briefs, https://ipad.fas.usda.gov/cropexplorer/ pecad_stories.aspx?regionid=br\&ftype=prodbriefs (August $15,2021)$.

Associate Editor: Marcia Pinheiro Margis

License information: This is an open-access article distributed under the terms of the Creative Commons Attribution License (type CC-BY), which permits unrestricted use, distribution and reproduction in any medium, provided the original article is properly cited. 